\title{
HIV-1 Capsid as a Target for Antiviral Therapy
}

Zhiqing Zhang', Feng Zhang ${ }^{1}$, Chao Yang ${ }^{1}$, Jialong Qi', Shuangquan Gao', Shaowei $\mathrm{Li}^{1,2}$, Ying Gu ${ }^{1,2 *}$ and Ningshao Xia ${ }^{1,2}$

${ }^{1}$ State Key Laboratory of Molecular Vaccinology and Molecular Diagnostics, School of Public Health, Xiamen University, Xiamen, China

${ }^{2}$ National Institute of Diagnostics and Vaccine Development in Infectious Disease, School of Life Sciences, Xiamen University, Xiamen, China

\begin{abstract}
Highly active anti-retroviral therapy (HAART) is the mainstay anti-HIV-1 therapy as it prolongs survival and switches HIV-1 infection from a fatal disease to a chronic yet manageable one. Unfortunately, drug toxicity and the emergence of drug-resistant mutant strains in patients undergoing long-term therapy have meant that there is still a continual need for novel drugs that target alternative molecules in the HIV-1 life cycle. The HIV-1 Gag precursor protein is a multidomain polyprotein that is proteolytically cleaved into the main, mature capsid protein; CA. CA has multifaceted roles during HIV-1 morphogenesis and is thus regarded as a promising target for future antiviral intervention. In this review, we describe the advances made in our understanding of the HIV-1 capsid structure and the key interactions involved during core assembly, and discuss how this and future knowledge will provide important structural insight for antiviral design.
\end{abstract}

Keywords: Human immunodeficiency virus; Capsid; Structure; Antivirals

\section{Introduction}

Human immunodeficiency virus type 1 (HIV-1) is the causative virus responsible for acquired immune deficiency syndrome (AIDS), for which there is still no protective vaccine. Since 1983, more than 34 million people have been infected with HIV across the world, with 1.5 million people dying as a result of complications from infection each year. HIV-1 virions mainly infect cells of the immune system and lead to a gradual reduction in the proportion of $\mathrm{CD} 4^{+} \mathrm{T}$ cells [1]. This in turn causes a significant decrease in the patient's ability to mount an immune response, and they die as a result of opportunistic infection. HIV-1 thus remains a major pathogenic threat to human health worldwide.

The HIV-1 replication cycle resembles that of other retroviruses, and this knowledge has helped to identify a range of potential targets for treatment. During the early stages of infection, when a viral particle is adsorbed to a target cell, the gp120 surface envelope (Env) glycoprotein binds to the CD4 receptor and either the chemokine receptor-5 (CCR5) or the CX chemokine receptor-4 (CXCR4) as a co-receptor [2]. Following this, the Env transmembrane glycoprotein gp41 undergoes a conformational change that promotes fusion of the viral and cellular membranes [3]. This process can be stalled by producing a neutralizing antibody that targets the Env glycoprotein. Likewise, fusion inhibitors can be developed to target the fusion process before infection. Fusion initiates a cascade of intracellular events comprising uncoating, reverse transcription, and integration, and each of these stages of infection offer important antiviral targets [4]; for example, the T20 inhibitor is the first FDA-approved fusion inhibitor for HIV-1/AIDS that works by binding to gp41 [4,5].

Another class of inhibitors is protease inhibitors, which mainly block the virus maturation process during the late phase, encompassing the events that result in virus gene expression, the assembly new virions, and the release and maturation of infectious virions [6]. Virus assembly and release are predominantly driven by the Gag polyprotein precursor $[7,8]$, whereas the maturation process is mediated by proteolysis events that trigger conformational changes to Gag, thereby converting immature particles to mature virions. In the immature particles, the Gag precursors assemble in a radial manner, whereas, in the mature particle, the viral protease liberates capsid CA proteins from the individual Gag domains, and these proteins re-assemble to form a conical capsid core referred to as the fullerene-like capsid [9].
There are more than two-dozen drugs approved for clinical use against HIV, and these drugs are reported to target various critical steps in the virus replication cycle [10]. Most drugs target the HIV-1 reverse transcriptase (RT) or protease (PR) enzymes, and, although they show marked efficacy in the short term, resistance develops rapidly against these drugs. In 1996, highly active antiretroviral therapy (HAART) was introduced as a combinatorial approach, and it has remained the mainstay for HIV-1 therapy. Patients are routinely prescribed two nucleoside RT inhibitors (NRTIs) along with a protease inhibitor (PI), a non-nucleoside RT inhibitor (NNRTI) or an integrase strand-transfer inhibitor (INSTI). Such regimens have turned HIV-1 infection from a lethal disease into a chronic one that can be managed. If adequately treated, patient life expectancy can be greatly extended. However, there are side effects with HAART, and the seemingly unavoidable emergence of drug-resistant mutant strains with long-term therapy [10]. Consequently, there is still a need for the development of novel drugs targeting other molecules in the HIV-1 life cycle.

The HIV-1 Gag precursor protein is a multi-domain polyprotein, and its predominant mature product, the CA capsid protein, plays a multifaceted role during HIV-1 morphogenesis and uncoating [11]. The advances in our understanding of the structure and function of CA have made it a promising target for future antiviral intervention [12]. In this review, we describe these major advancements and discuss the key interactions involved in core assembly and its structural basis for current antiviral design.

\section{The capsid protein, $\mathrm{CA}$}

Structurally, the Gag precursor protein is divided into four major domains: the N-terminal matrix (MA), the capsid (CA), the

*Corresponding author: Ying Gu, National Institute of Diagnostics and Vaccine Development in Infectious Disease, School of Life Sciences, Xiamen University, Xiamen, China, Tel: 8613850019366; E-mail: guying@xmu.edu.cn

Received November 25, 2015; Accepted January 06, 2016; Published January 15, 2016

Citation: Zhang Z, Zhang F, Yang C, Qi J, Gao S, et al. (2016) HIV-1 Capsid as a Target for Antiviral Therapy. J AIDS Clin Res 7: 536. doi:10.4172/21556113.1000536

Copyright: () 2016 Zhang Z, et al. This is an open-access article distributed under the terms of the Creative Commons Attribution License, which permits unrestricted use, distribution, and reproduction in any medium, provided the original author and source are credited. 
nucleocapsid (NC), and the C-terminal p6 (Figure 1A). Two spacer peptides are located between CA and NC (SP1) and between NC and p6 (SP2) $[8,13]$. CA is primarily responsible for the structure of both the immature Gag lattice and the mature viral core that undergoes conformational changes. The CA protein comprises 231 aa, and is a component of three proteins during its lifetime: the full-length p55 (Gag), the precursor $\mathrm{p} 41$, and $\mathrm{p} 25$, which are proteolytic intermediates.

The structures of CA and its isolated domains have been solved by X-ray crystallography and nuclear magnetic resonance (NMR) spectroscopy $[14,15]$. The monomer CA contains two independently folded domains-the $\mathrm{N}$-terminal domain and C-terminal domain (NTD and CTD, respectively), which are connected by a short flexible linker (Figure 1B). The NTD (CA residues 1-145) comprises seven a-helices (CA helices 1-7). Connecting helices 4 and 5 is a prolinerich loop that binds cyclophilin A $[16,17]$ Studies have shown that cyclosporine can disrupt the CA-cyclophilin A interaction and inhibit HIV-1 replication [18]. Similarly, a non-immunosuppressive analog of cyclosporine, Debio-025, can inhibit early-stage HIV-1 replication, with higher potency than that exhibited by cyclosporine [19].

The CTD (residues 151-231) is composed of a short 310 -helix followed by an extended strand and four $\alpha$-helices (CA helices 8-11). The CA CTD tends to dimerize through helix 9 on each monomer [20]. Besides, there is also a novel shoulder-to-shoulder CA dimerization mode mediated by an S-S bridge [21]. Mutations to residues 184 and 185 (W184A and M185A) can significantly reduce particle production in cells and CA dimerization in vitro [22,23]. In helix 8 , there is a highly conserved sequence known as the major homology region (MHR), which plays an important role in Gag assembly [22,23]. This MHR motif mediates conformational stabilization of CTD folding. The interface of MHR-swapped CTD [24] provides an anti-HIV target for designing compounds that inhibit capsid assembly.

\section{The immature HIV-1 capsid structure}

HIV-1 assembly proceeds at the plasma membrane when Gag, which is synthesized in the cytosol, trafficks to the site of assembly. The CA domain is the main viral determinant driving Gag assembly. The Gag protein packages the full-length genomic RNA into the nascent, immature virus particle together with the GagPol precursor. The structure of the intact, full-length Gag precursor is still unclear, owing to large size of Gag and the presence of flexible interdomain connections. Cryoelectron microscopy (cryo-EM) and cryoelectron tomography (cryo-ET) of the immature virus particle show that Gag molecules are

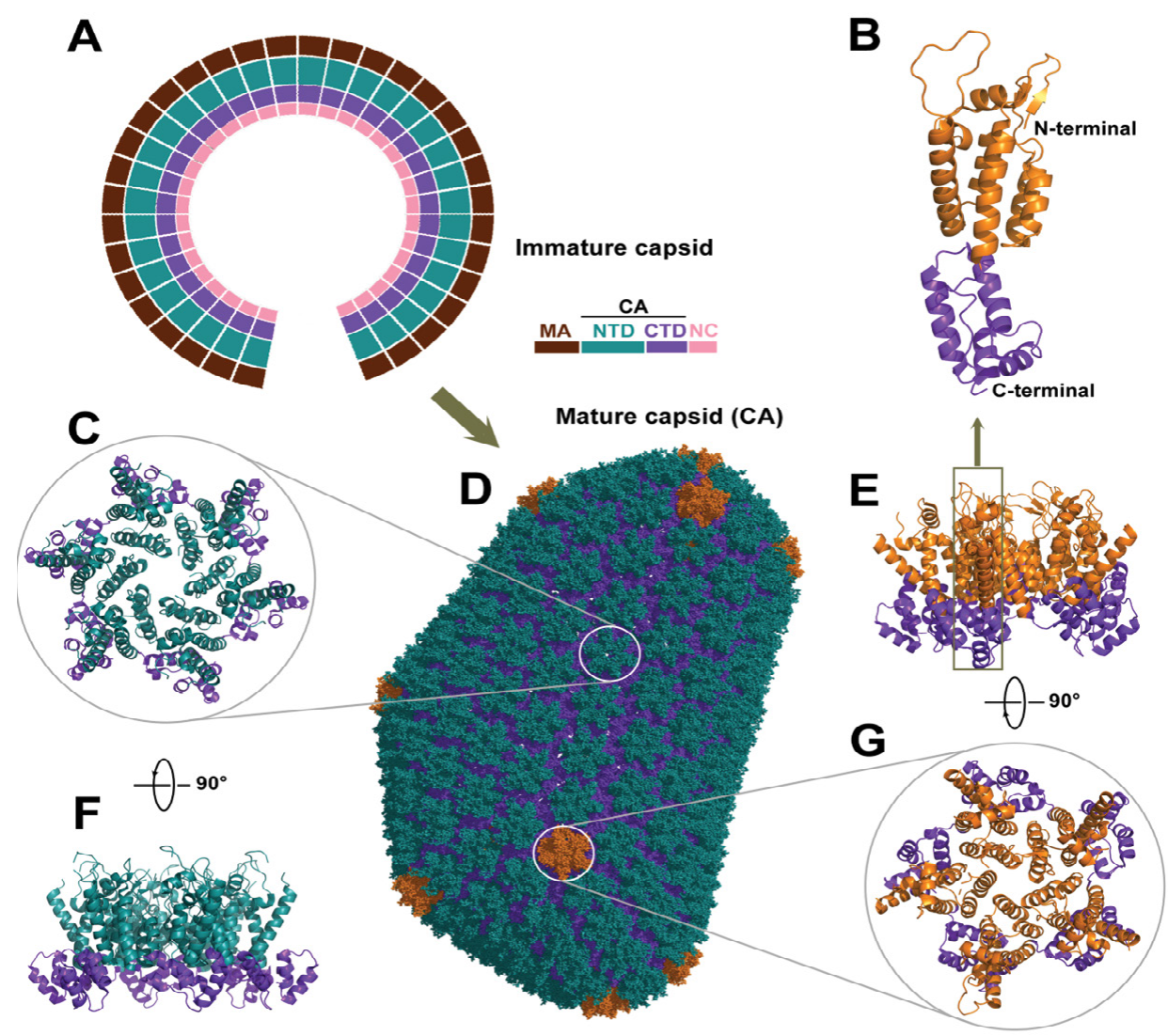

Figure 1: Structural delineation for HIV-1 CA proteins in various forms. (A) Cartoon model of immature capsid. HIV-1 Gag gene encodes the immature capsid, which is mainly comprised of MA (Matrix protein), CA (Capsid protein) and NC (Nucleo-capsid protein) in N- to C- terminal sequential arrangement, as shown in right-lower bar with same color-code with the model. The immature capsid was deemed unclosed globular shell, ready for rearrangement into mature version (CA). (B) Crystal structure of monomeric CA. The structure (PDB no. 2M8N) is rendered in cartoon mode, showing N-terminal domain (NTD) in orange and C-terminal domain (CTD) in purple-blue. (C) Crystal structure of hexameric CA. The structure (PDB no. 3H47) is rendered from top view of hexamer ring, with NTD in deep teal and CTD in purpleblue. (D) Structural model of mature capsid in terms of electron tomography and molecular dynamics. The structure (PDB no. 3J3Y) is rendered in line mode, exhibiting fullerene cone shape and containing 12 CA pentamers and 186 CA hexamers with NTD in orange and CTD in deep teal, respectively. One of pentamers boxed in white circles is further interpreted by crystal structure of pentamer $(G)$, one of hexamers by crystal structure in (C). (E) Crystal structure of pentameric CA. The structure (PDB no. 3P05) is rendered from side view of pentamer plane, with NTD in orange and CTD in purple-blue. (F) Side view of hexameric CA, related to (C) by $90^{\circ}$ horizontally inward rotation. $(\mathrm{G})$ Top view of pentameric $C A$, relating to $(E)$ by $90^{\circ}$ horizontally outward rotation. 
aligned and packaged radially (Figure 1A), with the MA domain bound to the inner leaflet of the viral membrane and the C-terminal region of Gag oriented toward the center of the particle [25].

The Gag subunits in the immature structure are organized as a lattice of hexamers, with a spacing of $8 \mathrm{~nm}$, and this arrangement forms an intermediate layer of cup-shaped structures, each made (from top to bottom) from a hollow hexameric ring of NTD domains, a hexameric ring of CTD domains, and a stem formed by six SP1 segments [26]. Previous structural analyses suggest that Gag hexamers are stabilized mainly by a six-helix bundle of SP1 peptides and CTD-CTD interactions, whereas the hexamers are linked to one another mainly through NTD-NTD and CTD-CTD homodimerization at interfaces. The immature Gag lattice is continuous but incomplete to accommodate the curvature necessary to form a particle with a diameter of $\sim 120 \mathrm{~nm}$.

Because the structure of the immature Gag lattice is curved and flexible, it has been technically more challenging to define. Recent breakthroughs in both the hardware and software algorithms required for cryo-EM and cryo-ET have facilitated improved HIV-1 structural analyses. Bharat et al. combined cryo-EM and cryo-ET and were able to solve the structure of the immature Gag lattice in in vitro-assembled tubes formed by a truncated version of the betaretrovirus MasonPfizer monkey virus (MPMV) Gag protein [27]. The 8- $\AA$ resolution structure permitted the derivation of a pseudo-atomic model of CA in the immature retrovirus, and this led to the discovery that CA-CA contacts in the immature Gag lattice differ from those in the mature CA lattice. Later work by the group revised the MPMV-based model by applying cryo-ET and sub-tomogram averaging methods to resolve the structure of the capsid lattice within intact immature HIV-1 particles at subnanometre resolution [28]. The resulting model demonstrates that the quaternary structural interactions mediating HIV-1 assembly differ substantially from that of the MPMV CA proteins. Although the observed electron densities show the SP1 assembles a six-helix bundle consistent with previous models, the precise structure of SP1 is not resolved. Early studies provided evidence that the CA-SP1 cleavage site is the binding site for HIV-1 maturation inhibitors. Bevirimat (BVM), which blocks the CA-SP1 cleavage site [29], is the first Gagtargeted compound to reach clinical trials (Table 1); however, its exact

\begin{tabular}{|c|c|c|c|c|c|c|c|}
\hline Antiviral reagent & Structure/sequence & $\begin{array}{l}\text { PDB no. of co- } \\
\text { crystal structure }\end{array}$ & $\begin{array}{l}\text { Binding pocket } \\
\text { localized in CA }\end{array}$ & $\begin{array}{l}\text { Target residues for } \\
\text { antiviral function }\end{array}$ & Antiviral mechanism & $\begin{array}{l}\text { R\&D } \\
\text { stage }\end{array}$ & References \\
\hline $\begin{array}{l}\text { Bevirimat } \\
\text { (BVM) }\end{array}$ & & $\begin{array}{l}\text { Hypothetical } \\
\text { binding model } \\
\text { based on PDB } \\
\text { no. } 1 \cup 57\end{array}$ & $\begin{array}{l}\text { The CA-SP1 } \\
\text { cleavage site }\end{array}$ & $\mathrm{N} / \mathrm{A}$ & $\begin{array}{l}\text { Stabilize immature } \\
\text { Gag lattice and block } \\
\text { HIV-1 maturation }\end{array}$ & Phase II & {$[29,30]$} \\
\hline CAP-1 & & 2JPR & $\begin{array}{l}\text { Helices } 1,2,4 \\
\text { and } 7\end{array}$ & $\begin{array}{l}\text { W23, V27, V31, } \\
\text { F32, V59, V65, } \\
\text { M66, I138, I141 }\end{array}$ & $\begin{array}{l}\text { Disrupt HIV-1 } \\
\text { assembly }\end{array}$ & Preclinical & [39] \\
\hline CAI & ITFEDLLDYYGP & 2BUO & $\begin{array}{l}\text { Helices } 8,9 \text { and } \\
11\end{array}$ & $\begin{array}{l}\text { D166, Y169, K182, } \\
\text { N183, E187, E212, } \\
\text { M215 }\end{array}$ & $\begin{array}{c}\text { Disturb CA dimerization } \\
\text { and disrupt HIV-1 } \\
\text { assembly }\end{array}$ & Preclinical & [41] \\
\hline PF74 & & $\begin{array}{l}4 \mathrm{UOE} \\
2 \mathrm{XDE}\end{array}$ & $\begin{array}{c}\text { Helices } 3,4,5 \text { and } \\
\text { 7; Indole interacts } \\
\text { with CTD }\end{array}$ & $\begin{array}{c}\text { N57, Q63, Q67, } \\
\text { K70, I73, T107, } \\
\text { L172, R173 }\end{array}$ & $\begin{array}{l}\text { Affect capsid } \\
\text { stability; Inhibit HIV-1 } \\
\text { replication in early } \\
\text { and late phases }\end{array}$ & Preclinical & [12] \\
\hline $\begin{array}{l}\text { Benzodiazepines } \\
\text { (BD3) }\end{array}$ & & 4E91 & $\begin{array}{l}\text { Helices 1, 2, } 4 \\
\text { and } 7\end{array}$ & V24, F32, V59, H62 & $\begin{array}{l}\text { Inhibit immature } \\
\text { capsid assembly }\end{array}$ & Preclinical & {$[40]$} \\
\hline $\begin{array}{l}\text { Benzimidazoles } \\
\text { (BM4) }\end{array}$ & & 4E92 & $\begin{array}{l}\text { Helices 1, 2, } 4 \\
\text { and } 7\end{array}$ & $\mathrm{~F} 32, \mathrm{H} 62$ & $\begin{array}{l}\text { Prevent capsid } \\
\text { maturation }\end{array}$ & Preclinical & [40] \\
\hline $\mathrm{BI}-1$ & & 4J93 & $\begin{array}{l}\text { Helices } 3,4,5 \\
\text { and } 7\end{array}$ & $\begin{array}{l}\text { N53, L56, N57, } \\
\text { K70, I73, A105, } \\
\text { G106, T107 }\end{array}$ & $\begin{array}{l}\text { Inhibit nuclear import } \\
\text { in early phase of HIV- } \\
1 \text { replication }\end{array}$ & Preclinical & [47] \\
\hline
\end{tabular}

Table 1: Structure, antiviral mechanism and research stage of antiviral reagents targeting to HIV-1 CA summarized in this review. 
mechanism of action is not well-defined [30]. Further studies will be required to obtain the structure of the CA-SP1 boundary region in the context of the assembled, immature Gag, and this insight will help to determine the precise location of the Bevirimat binding site.

\section{The mature HIV-1 capsid structure}

The mature capsid structure is well-defined. During protease processing of the mature Gag, the capsid undergoes a major change in virion morphology. Unlike the immature structure, the CA subunits in the mature structure are organized as a lattice of hexamers with $10-\mathrm{nm}$ spacing. As mentioned earlier, the mature capsid resembles a fullerene cone, with the hexameric lattice closed off by seven pentamers at its wide end and five at its narrow end (Figure 1D) [31]. There are three different interfaces for the CA-CA interaction in the mature capsid: (i) the NTD-NTD interface between NTD domains in the hexamers; (ii) the NTD-CTD interface between the NTD and CTD domains belonging to neighboring subunits of the same hexamer; (iii) the CTD-CTD interface between CTD domains belonging to neighboring hexamers. These three inter-subunit interfaces have been resolved at the atomic level by X-ray crystallography and cryo-EM $[15,32]$.

HIV-1 CA spontaneously polymerizes into capsid-like particles in vitro, and therefore it does not form hexamers or pentamers in solution. To overcome this problem, Pornillos et al. introduced disulfide bridges at the NTD-NTD interface to crosslink the monomers and stabilize the hexamer (Figures $1 \mathrm{C}$ and $1 \mathrm{~F}$ ) or pentamer (Figures $1 \mathrm{E}$ and $1 \mathrm{G}$ ). They also introduced mutations to two residues-W184A and M185A-at the CTD-CTD interface to weaken the CA-CA dimerization [33-35]. The group then went on to solve the atomic structures of the hexamers and pentamers by X-ray crystallography. Zhao et al reported the mature HIV-1 capsid structure at 8 - $\AA$ resolution by cryo-EM and using allatom molecular dynamics (Figure 1D) [36]. The resolved structure showed a three-helix bundle with critical hydrophobic interactions at the CTD trimer interface; further mutation analysis indicated that this bundle played a critical role in the assembly and stability of the mature capsid. The complete atomic HIV-1 capsid model provided a basis to further study capsid function and identifies targets that were appropriate for pharmacological intervention.

More recent X-ray crystallography findings of the native HIV-1 capsid protein have revealed conformational variability [37]. This higherresolution native CA structure demonstrates interactions between CA monomers, with six-fold symmetry within hexamers (intrahexamer), and three- and two-fold symmetry between neighboring hexamers (inter-hexamer). The results also show hydrophilic, water-mediated interactions, the disruption of which alters these inter-hexameric interfaces. These new insights bring a clearer picture of HIV-1 biology and will aid in the design of better antiretroviral drugs. However, the HIV-1 assembly mechanism itself remains unclear, and future
A

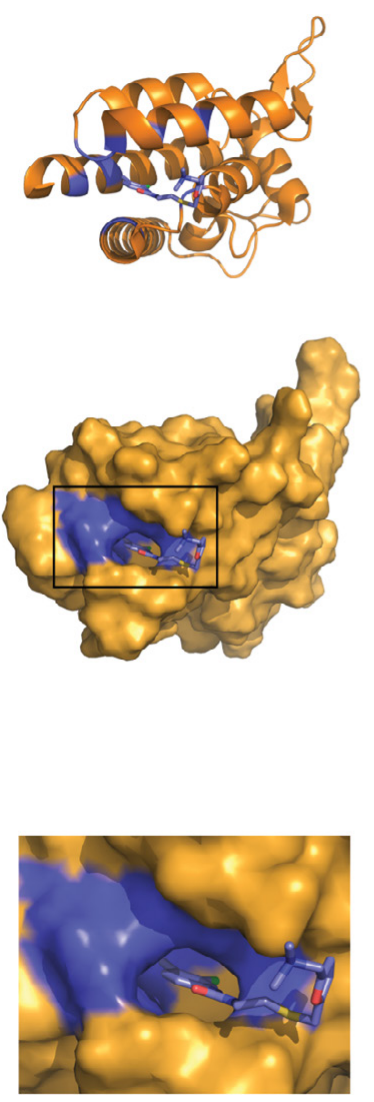

B
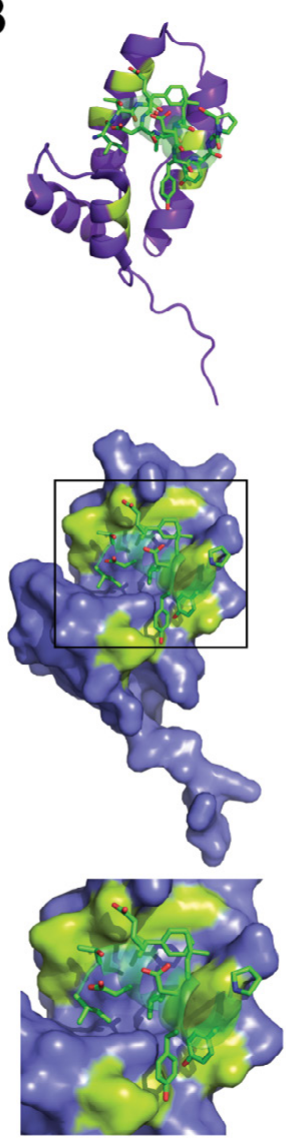

C
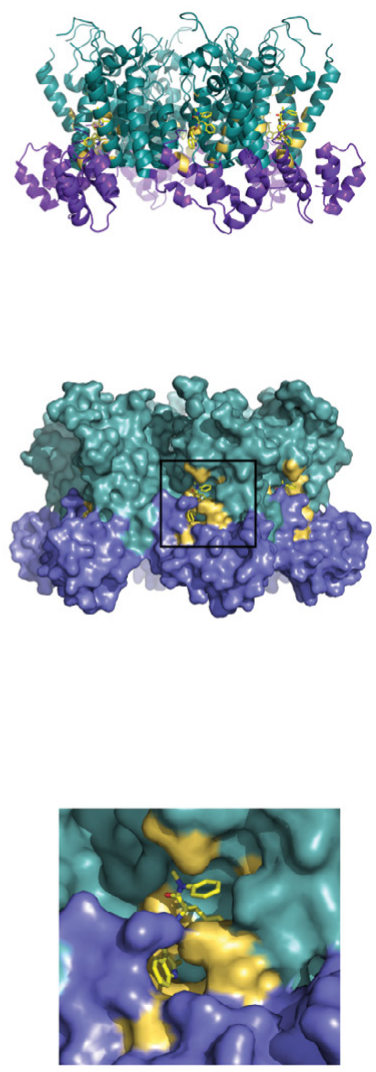

Figure 2: Co-crystal structures of $C A$ proteins binding with 3 representative antiviral reagents. Inhibitor CAP-1 (A), CAI (B) and PF74 (C) in stick view bind with CA proteins in co-crystal structures (PDB nos. 2JPR, 2BUO and 4U0E, respectively). Top panels, inhibitors shown in atomic color-code stick mode, CA rendered in cartoon view with target residues (referred to Table 1 ) colored as $C$ atom of inhibitor; Middle panels, same as top ones, while CA rendered in surface view; Bottom panels, closed-up views for the rectangle boxes in Middle panels to show the binding pockets of the inhibitors. 
work should be aimed at determining how the immature CA lattice disassembles and reassembles to form the mature CA lattice.

\section{CA as a target of antivirals}

The HIV-1 capsid protein plays a central role in the virus replication cycle, making it an attractive target for the development of antiHIV-1 inhibitors (Table 1). Disruption of CA-CA contacts by specific compounds could be used to block the assembly of immature particles or the mature capsid core. An early small-molecule inhibitor, CAP-1, was identified by Summers and colleagues with an in silico screening approach [38]. NMR and X-ray crystallographic studies showed that CAP-1 binds to a pocket formed at the point at which helices 1, 2, 4 and 7 of the NTD interact (Figure 2A) [39]. This interaction disrupts virus assembly and maturation, and this pocket is also the binding site for other small molecules, such as $\beta$-defensin 3 and benzimidazole-4 [40].

The CTD-binding peptide CAI (ITFEDLLDYYGP) was screened by phage display. This peptide inhibits both immature and mature capsid assembly in vitro. Structure analysis of the CAI-CTD complex showed that the peptide binds to a hydrophobic groove formed by CA helices 8, 9 and 11 (Figure 2B) [41]. CAI binding to the CTD causes steric interference, limiting the formation of the native NTD-CTD interface between CA subunits in the mature capsid hexamers. Furthermore, the binding also induces a conformational rearrangement of the CTD dimer, which destabilizes the CTD dimerization interface between hexamers [42]. To improve the affinity of CAI for the CTD and to enhance its cellpenetrating ability, CAI derivatives were developed using hydrocarbon stapling. Two of these derivatives, NYAD-1 (ITFXDLLXYYGKKK) and NYAD-13 (ITFLDLLLYYGKKK), have been shown to inhibit HIV-1 infection ex vivo and display a broad spectrum of antiviral activity [43].

The compound PF-3450074 (also known as PF74) is the bestcharacterized small-molecule inhibitor targeting the viral CA protein [12]. Structure analyses indicate that PF74 makes contacts with the HIV-1 CA hexamer at the NTD-CTD interface, binding with higher affinity to the intact hexamer than to monomeric CA. The R1 and R2 aromatic groups of PF74 occupy the hydrophobic pocket formed by HIV-1 CA-NTD helices 3, 4, 5 and 7. Meanwhile, the R3 indole interacts with CTD in the hexamer-bound form (Figure 2C). Blair and colleagues speculated that PF74 specifically targets the assembled capsid, rather than the unassembled CA subunit. PF74 exerts inhibition over normal capsid assembly and disassembly in the early and late phasess of HIV infection. PF74 was found to accelerate CA assembly in HIV-1 CA multimerization assay [12]. However, the treatment over viral cores isolated from HIV-1 virion/pseduoviron with PF74 can destabilize the mature capsid [44]. The findings imply that PF74 might function in different events of CA assembly or disassembly throughout the virus life cycles.

Recent studies have uncovered the more nuanced mechanism of action of this compound: (1) At higher concentrations, PF74 destabilizes viral core. And at lower concentrations, potent inhibition is retained because it competes with the binding of two host factors, nuclear pore complex protein 153 (NUP153) and cleavage and polyadenylation specificity factor 6 (CPSF6) [45]. (2) The reported cocrystal structure shows PF74 targets the same binding pocket in the assembled CA as those of CPSF6 and NUP153 [46]. Thus, PF74 might implement antiviral activity by blocking CA from binding with the two host factors. (3) A new crystal structure of native CA suggests that PF74 might affect the capsid stability by inducing subtle changes at the interhexamer interfaces [37].

The Boehringer Ingelheim group identified two classes of compounds, benzodiazepines (BD) and benzimidazoles (BM) [40]. BD and BM target to CA NTD with the binding sites similar as that of CAP1 , and otherwise exhibit higher antiviral activity. Another compound named BI-1 interacts with CA NTD on binding sites overlapping with that of PF74, but presents distinct functional mode in the antiviral research [47]. All the antiviral reagents mentioned in this review were listed in Table 1 with the information of structure, antiviral mechanism and research stage.

\section{Perspective}

Although numerous inhibitors targeting the capsid have been identified to date, they have not yet to be successfully exploited in clinically approved antiviral therapies. It is likely that resistance to the current anti-HIV-1 drugs will become an increasing problem in the near future, and this will necessitate the development of novel drugs. We believe that the HIV-1 capsid will become a promising target because of its important function in the HIV-1 life cycle. In recent years, we have witnessed remarkable advances in our understanding of HIV-1 immature and mature capsid structures, and we have gained insight into the important function of the capsid protein in the replication cycle. However, many questions remain to be fully answered. For example, what is the structure of the CA-SP1 boundary region in the assembled Gag lattice, and how do maturation inhibitors bind to this region? How does the immature CA lattice disassemble and reassemble to form the mature CA lattice? Exploring the structure-function relationship of the capsid in answering these questions will help to drive CA-targeted therapeutic opportunities.

\section{Acknowledgement}

The work was supported by National Natural Science Foundation (Grant no. 81371818).

\section{References}

1. Swanstrom R, Coffin J (2012) HIV-1 pathogenesis: the virus. Cold Spring Harb Perspect Med 2: a007443.

2. Freed EO (2001) HIV-1 replication. Somat Cell Mol Genet 26: 13-33.

3. Wilen CB, Tilton JC, Doms RW (2012) HIV: cell binding and entry. Cold Spring Harb Perspect Med 2.

4. Fassati $A$ (2012) Multiple roles of the capsid protein in the early steps of HIV-1 infection. Virus Res 170: 15-24.

5. Chong H, Qiu Z, Su Y, Yang L, He Y (2015) Design of a highly potent HIV-1 fusion inhibitor targeting the gp41 pocket. AIDS 29: 13-21.

6. Freed EO (2015) HIV-1 assembly, release and maturation. Nat Rev Microbio 13: 484-496.

7. Ganser-Pornillos BK, Yeager M, Sundquist WI (2008) The structural biology of HIV assembly. Curr Opin Struct Biol 18: 203-217.

8. Bell NM, Lever AM (2013) HIV Gag polyprotein: processing and early vira particle assembly. Trends Microbiol 21: 136-144.

9. Ganser-Pornillos BK, Yeager M, Pornillos O (2012) Assembly and architecture of HIV. Adv Exp Med Biol 726: 441-465.

10. Arts EJ, Hazuda DJ (2012) HIV-1 antiretroviral drug therapy. Cold Spring Harb Perspect Med 2: a007161.

11. Campbell EM, Hope TJ (2015) HIV-1 capsid: the multifaceted key player in HIV1 infection. Nat Rev Microbiol 13: 471-483.

12. Blair WS, Pickford C, Irving SL, Brown DG, Anderson M, et al. (2010) HIV capsid is a tractable target for small molecule therapeutic intervention. PLoS Pathog 6: e1001220

13. Tedbury PR, Freed EO (2015) HIV-1 gag: an emerging target for antiretroviral therapy. Curr Top Microbiol Immunol 389: 171-201.

14. Ganser-Pornillos BK, Cheng A, Yeager M (2007) Structure of full-length HIV-1 CA: a model for the mature capsid lattice. Cell 131: 70-79. 
15. Byeon I-JL, Meng X, Jung J, Zhao G, Yang R, et al. (2009) Structural convergence between Cryo-EM and NMR reveals intersubunit interactions critical for HIV-1 capsid function. Cell 139: 780-790.

16. Gamble TR, Vajdos FF, Yoo S, Worthylake DK, Houseweart M, et al. (1996) Crystal structure of human cyclophilin A bound to the amino-terminal domain of HIV-1 capsid. Cell 87: 1285-1294.

17. Cortines JR, Lima LM, Mohana-Borges R, Millen Tde A, Gaspar LP, et al. (2015) Structural insights into the stabilization of the human immunodeficiency virus type 1 capsid protein by the cyclophilin-binding domain and implications on the virus cycle. Biochim Biophys Acta 1854: 341-348.

18. Luban J (2007) CyclophilinA, TRIM5, and resistance to human immunodeficiency virus type 1 infection. J Virol 81: 1054-1061.

19. Daelemans D, Dumont JM, Rosenwirth B, De Clercq E, Pannecouque C (2010) Debio-025 inhibits HIV-1 by interfering with an early event in the replication cycle. Antiviral Res 85: 418-421.

20. Ivanov D, Tsodikov OV, Kasanov J, Ellenberger T, Wagner G, et al. (2007) Domain-swapped dimerization of the HIV-1 capsid C-terminal domain. Proc Natl Acad Sci U S A 104: 4353-4358.

21. Gu Y, Cao F, Wang L, Hou W, Zhang J, et al. (2013) Structure of a nove shoulder-to-shoulder p24 dimer in complex with the broad-spectrum antibody A10F9 and its implication in capsid assembly. PLoS One 8: e61314.

22. Purdy JG, Flanagan JM, Ropson IJ, Rennoll-Bankert KE, Craven RC (2008) Critical role of conserved hydrophobic residues within the major homology region in mature retroviral capsid assembly. J Virol 82: 5951-5961.

23. Chang YF, Wang SM, Huang KJ, Wang CT (2007) Mutations in capsid major homology region affect assembly and membrane affinity of HIV-1 Gag. J Mol Biol 370: 585-597.

24. Bocanegra R, Fuertes MÁ, Rodríguez-Huete A, Neira JL, Mateu MG (2015) Biophysical analysis of the MHR motif in folding and domain swapping of the HIV capsid protein C-terminal domain. Biophys J 108: 338-349.

25. Briggs JA, Riches JD, Glass B, Bartonova V, Zanetti G, et al. (2009) Structure and assembly of immature HIV. Proc Natl Acad Sci U S A 106: 11090-11095.

26. Wright ER, Schooler JB, Ding HJ, Kieffer C, Fillmore C, et al. (2007) Electron cryotomography of immature HIV-1 virions reveals the structure of the CA and SP1 Gag shells. EMBO J 26: 2218-2226.

27. Bharat TA, Davey NE, Ulbrich P, Riches JD, de Marco A, et al. (2012) Structure of the immature retroviral capsid at $8 \AA$ resolution by cryo-electron microscopy. Nature 487: 385-389.

28. Schur FK, Hagen WJ, Rumlová M, Ruml T, Müller B, et al. (2015) Structure of the immature HIV-1 capsid in intact virus particles at $8.8 \AA$ resolution. Nature 517: 505-508.

29. Nguyen AT, Feasley CL, Jackson KW, Nitz TJ, Salzwedel K, et al. (2011) The prototype HIV-1 maturation inhibitor, bevirimat, binds to the CA-SP1 cleavage site in immature Gag particles. Retrovirology 8: 101.

30. Keller PW, Adamson CS, Heymann JB, Freed EO, Steven AC (2011) HIV-1 maturation inhibitor bevirimat stabilizes the immature Gag lattice. J Virol 85: $1420-1428$.

31. Briggs JA, Grünewald K, Glass B, Förster F, Kräusslich HG, et al. (2006) The mechanism of HIV-1 core assembly: insights from three-dimensional reconstructions of authentic virions. Structure 14: 15-20.

32. Mateu MG (2009) The capsid protein of human immunodeficiency virus: intersubunit interactions during virus assembly. FEBS J 276: 6098-6109.
33. Pornillos O, Ganser-Pornillos BK, Kelly BN, Hua Y, Whitby FG, et al. (2009) X-ray structures of the hexameric building block of the HIV capsid. Cell 137 1282-1292.

34. Pornillos O, Ganser-Pornillos BK, Banumathi S, Hua Y, Yeager M (2010) Disulfide bond stabilization of the hexameric capsomer of human immunodeficiency virus. J Mol Biol 401: 985-995.

35. Pornillos O, Ganser-Pornillos BK, Yeager M (2011) Atomic-level modelling of the HIV capsid. Nature 469: 424-427.

36. Zhao G, Perilla JR, Yufenyuy EL, Meng X, Chen B, et al. (2013) Mature HIV-1 capsid structure by cryo-electron microscopy and all-atom molecular dynamics. Nature 497: 643-646

37. Gres AT, Kirby KA, KewalRamani VN, Tanner JJ, Pornillos O, et al. (2015 STRUCTURAL VIROLOGY. X-ray crystal structures of native HIV-1 capsid protein reveal conformational variability. Science 349: 99-103.

38. Tang C, Loeliger E, Kinde I, Kyere S, Mayo K, et al. (2003) Antiviral inhibition of the HIV-1 capsid protein. J Mol Biol 327: 1013-1020.

39. Kelly BN, Kyere S, Kinde I, Tang C, Howard BR, et al. (2007) Structure of the antiviral assembly inhibitor CAP-1 complex with the HIV-1 CA protein. J Mol Biol 373: 355-366.

40. Lemke CT, Titolo S, von Schwedler U, Goudreau N, Mercier JF, et al. (2012) Distinct effects of two HIV-1 capsid assembly inhibitor families that bind the same site within the N-terminal domain of the viral CA protein. J Virol 86: 66436655.

41. Ternois F, Sticht J, Duquerroy S, Kräusslich HG, Rey FA (2005) The HIV-1 capsid protein $\mathrm{C}$-terminal domain in complex with a virus assembly inhibitor Nat Struct Mol Biol 12: 678-682.

42. Barklis E, Alfadhli A, McQuaw C, Yalamuri S, Still A et al. (2009) Characterization of the in vitro HIV-1 capsid assembly pathway. J Mol Biol 387: 376-389.

43. Zhang H, Zhao Q, Bhattacharya S, Waheed AA, Tong X, et al. (2008) A cellpenetrating helical peptide as a potential HIV-1 inhibitor. J Mol Biol 378: 565580 .

44. Shi J, Zhou J, Shah VB, Aiken C, Whitby K (2011) Small-molecule inhibition of human immunodeficiency virus type 1 infection by virus capsid destabilization. J Virol 85: 542-549.

45. Price AJ, Jacques DA, McEwan WA, Fletcher AJ, Essig S, et al. (2014) Host cofactors and pharmacologic ligands share an essential interface in HIV-1 capsid that is lost upon disassembly. PLoS Pathog 10: e1004459.

46. Bhattacharya A, Alam SL, Fricke T, Zadrozny K, Sedzicki J, et al. (2014 Structural basis of HIV-1 capsid recognition by PF74 and CPSF6. Proc Natl Acad Sci U S A 111: 18625-18630.

47. Lamorte L, Titolo S, Lemke CT, Goudreau N, Mercier JF, et al. (2013) Discovery of novel small-molecule HIV-1 replication inhibitors that stabilize capsid complexes. Antimicrob Agents Chemother 57: 4622-4631. 